\title{
Author Correction: The interplay between membrane lipids and phospholipase A family members in grapevine resistance against Plasmopara viticola
}

Gonçalo Laureano $\mathbb{D}^{1}$, Joana Figueiredo $\mathbb{D}^{1,2,3}$, Ana Rita Cavaco ${ }^{1}$, Bernardo Duarte $\mathbb{D}^{4}$, Isabel Caçador ${ }^{4}$, Rui Malhó ${ }^{1}$, Marta Sousa Silva ${ }^{2,3}$, Ana Rita Matos ${ }^{1}$ \& Andreia Figueiredo ${ }^{1}$

Correction to: Scientific Reports https://doi.org/10.1038/s41598-018-32559-z, published online 28 September 2018

This Article contains an error in the order of the Figures. Figures 6 and 7 were published as Figures 7 and 6 respectively. The Figure legends are correct and the correct Figures 6 and 7 appear below as Figures 1 and 2.

In addition, this Article contains a typographical error in the Results and Discussion section, under the subheading 'Lipid modulation during first hours of grapevine-P. viticola interaction' where,

"The proportions of PC and PG, the main phospholipid classes of leaf cell membranes, remain unchanged at 6 hpi (Fig. 3E; Supplementary Table S2)".

should read:

"The proportions of PC and PG, the main phospholipid classes of leaf cell membranes, remain unchanged at 6 hpi (Fig. 3D; Supplementary Table S2)". 
A) $\mathrm{VviPLA}_{1}-\mathrm{I} \alpha$ VviPLA $_{1}-\mathrm{I} \beta 1$ VviPLA $_{1}$-I $\beta 2$ VviPLA $_{1}-\mathrm{I} \beta 2$ VviPLA $_{1}-\mathrm{I} \gamma 1$ VviPLA $_{1}$-I 2 $\mathrm{VviPLA}_{1}$-II $\gamma 1 \mathrm{a}$ VviPLA - II $\gamma_{2}$ VviPLA - III VviPLA $_{1}$-III 1 $\mathrm{VviPLA}_{1}$-III 22

VviPLA $_{1}$-III $\beta 1$ a GEQVSLT ITGHSLGGALALLNAYEAATSL - - -
VviPLA $_{1}$-III 1 1b GEQVSLT ITGHSLGG$$
470
$$

徒 PLLIT ITGHSLAALA ILTAYDIKTTF - . GETLS ITVTGHSLGAALAVLVADELSTCD - DEQLS IT ITGHSLGAALATLAAYDIATTF ... NEELS ITFTGHSLGGALAVLSAYDVAETGLNV DEE IS IT ITGHSLGAALALLSAYDIAEMNLNV EEE IS ISLTGHSLGAAVATLNAVDIVANGLN DEE I I ISTTHSLGAAVATLNAVDIVANGLNDEEISITITGHSLGAALGTLCAADIVANKFNK

480

B) VviPA-PLA ${ }_{1}-\alpha$ GYDGKVS IYGHSLGSVLSYIII LCHQDNLSSPFP VviPA-PLA $1-\beta \quad$ GYDGKVSIYGHSLGSVLSYSILCHQDNLSSPFP GxSxG

C) $\mathrm{VvisPLA}_{2}$ 50 NCNSVG IRYGG' GGVG'WTGCPGE RYGKYCGXXXxGC 70 80 90 100

D) VvipPLA-I $\alpha$ VvipPLA-I $\beta$ VvipPLA-II $\alpha 1 b$ VvipPLA-II 22 VvipPLAA-II $\alpha 3$ VvipPLA-Il 23 VvipPLA-IIB1b VvipPLA-IIB2 VvipPLA-IIß2b

$530 \quad 540,550,560,570,580$ LDACCxxHDxCV

LR I L SMDGGG -MKGLG'TVQVLKE IEK - . - . - - - G - TGKR I HELFDL I CGTSTGGML LR ILSMDGGG - MKG LGTVQVL KE IEK-...- - - - TGKR I HELFDL I CGTSTGGML IT ILS IDGGG - IRGL IPGTVLGFLESELQKLDG - EDAR ISDYFDV I AGTSTGGLV IT I LS I DGGG - IRGL IPGTVLGFLESELQKLDG - EDAR ISDYFDV I AGTSTGGLV IPGTVLGFLESELQKLDG - EDAR ISDYFDV IAGTSTGGLV IT I ITILSIDGGG - IRGL IPGTVLGFLESELQKLDG -EDAR ISDYFDVIAGTSTGGLV VTILS IDGGG - IRG I I PAT ILALLESQLQELDG - DDAR IADYFDV I AGTSTGGLV VT ILS I DGGG - IRG I I PAT I LALLESQLQAVDG - DDAR I ADYFDV I AGTSTGGLV VT IFS IDGGG - IRG I IPAT ILARLETRLQELDG - DDAR I ADYFDV IAGTSTGG IV VT ILS IDGGG - IRG I IPAT ILARLETRLQELDG - DDAR I ADYFDV I AGTSTGG IV VT ILS I DGGG - IRG I IPAT I LACLESHLOELDG - DDAR I ADYFDV I AGTSTGGLV VT ILS IDGRG IRG IPAT ILACLESQLQELDG -DDARIADYFDVIAGTSTGGLV IT ILS I DGGG - VRG I I PAV I LSALEAQLQRLDG - PDAR I ADYFDL I AGTSTGS IV IT ILS I DGGG - VRG I IPAV I LSALEAELQRLDG - PDAR IADYFDL IAGTSTGS IV IT I LS I DGGG - VRG I IPAV ILSALEAQLQR IDG - PNAR I ADYFDV I AGTNTGS IV IT I LS I DGGG - VRG I ISG I I LASLESELQKLDG - EDARLSDYFDV I AGTSNGGL I ITVLS I DGGG - I KG I IPG I ILDFLESELQKLDG - EDARLADYFDVVSGTSTGGL I IT ILS I DGGG - VRG I IPG I ILAFLESELQKLDG - EDARLADYFDV I AGTSTGGL I VTVLS IDGGG - VRG I I PGTLLAFLESKFQELDG - DDAR I ADYFDV I AGTSTGGLV ITVLSMDGGG - IRG ILPGT ILSFLESKLQELDG - ADAR IADYFDV IAGTSTGGL I VA ILS I DGGGATDG I LAARSLAHLEASLRHKSGNPNAR I SDYFDVVAGSGAGG I L IC ILS I DGGG - MRG I LSGRALAYLEQALKTKSGNPQAR I ADYFDVAAGAGVGG IF TRVLS IDGGG - TTAVVATAALVHLEDQ IQLKTGDSHSR IVDFFDVVVGTG IGAVF DGGGxRG

800 810

GTSTG

VvipPLA-Io

VvipPLA-I $\beta$

VvipPLA-II $\alpha 1 \mathrm{a}$
VvipPLA-II $\alpha 1 \mathrm{~b}$ A IRASSAAPYYLDDFS - . - DDM - NRW - . - DDGA IVANNPTVFSMREA VvipPLA-II $\alpha 2$ ICIGTSAAPTYLPAHYFETKDPA-GRVREFNL I DGGVAANNPTLVA IGEV VvipPLA-II $\alpha 3$ IC IGTSAAPTYLPAHYFETKDPT - GRVREFNL I DGGVAANNPALVA IGEV VvipPLA-II $\beta 1$ I IC IGSSAAPTYFPGHYFKNQDKE - GKTQEFNL I DGGVAANNPALVA ISQV VvipPLA-II 1 Ib I C IGSSAAPTYFPGHYF KNQDKE - GKTQEFNL I DGGVAANNPAL VA ITQV VvipPLA-II $\beta 2 \mathrm{a}$ I C IGSSAAPTYFPAYFFKNQDKE - GKTQEFDL I DGGVAANNPALVA ITQV VvipPLA-IIB2b IC IGSSAAPTYFPAYFFKNQDKE - GKTOEFDL I DGGVAANNPALVAITQV $\begin{array}{ll}\text { VvipPLA-II } \beta 2 b & \text { I C IGSSAAPTYFPAYFF KNQDKE - GKTQEFDL I DGGVAANNPALVA ITQV } \\ \text { VvipPLA-II } 33 & \text { I C IGSSAAPTYFPAYYFKNQDKE - GKTQEFDL I DGGVAANNPALVA ITQV }\end{array}$ VvipPLA-II $\beta 4$ a IC IGSSAAPTYFPAYFFKNQDKE - GKARELNL IDGGVAANNPALVA ISQV VvipPLA-II $\beta 4$ b ICIGSSAAPTYFPAYFFKNQDKE - GKARELNL I DGGVAANNPALVA ISQV VVipPLA-II $\gamma 1$ a VVMSSAAAP VFFPSHNFE - - - AD - G - - RMYNL VDGGVAANNPTLLA IQEA VVipPLA-II $\gamma 1 b$ VVMSSAAAP VYFPSHNFK - - AD - G - RMYNLVDGGVAANNPTLLA IQEA VvipPLA-II $\gamma 2$ AVLGSAAAPISFRCHHFE - - AD - - - KIYNLVDGGMGANNPTLLA IREA VvipPLA-II $\delta$ ICIGTSAAPTYLPAYCFVNQDDQ-GSTREFNLIDGGLAASNPTLVA ISEV $\begin{array}{ll}\text { VvipPLA-II } \delta 1 & \text { IC IGTSAAPTYLPAYCF VNQDDQ - GSTREFNL IDGGLAASNPTLVA ISEV } \\ \text { VvipPLA-II } \delta 2 & \text { ICISTSAAPTFLPAYYFENPDEKGGKMREFNLTDGGVAANNPTLLA ISEV }\end{array}$ $\begin{array}{ll}\text { VvipPLA-II } 2 & \text { I C ISTSAAPTFLPAYYFENPDEKGGKMREFNLTDGGVAANNPTLLA ISEV } \\ \text { VvipPLA-II } 33 & \text { IC IGTSAAPTYLPAHYFENQDDQ - ENNEEFNL I DGGLAANNPCLVA ISEV }\end{array}$ $\begin{array}{ll}\text { VvipPLA-II } 3 & \text { IC IGTSAAPTYLPAHYFENQDDQ - ENNEEFNL IDGGLAANNP CLVA ISEV } \\ \text { VvipPLA-II } 1 & \text { IC ISTSAAPTYLPAHYFETKEAN - GKTRSFNL I DGGVAANNPTLVAMSH I }\end{array}$ VvipPLA-IIE2 IC ISTSSAPTYLPAYNFRTHDSD - GNEREFHLVDGGVAANNPALLAMKPT VVipPLA-III $\alpha$ VCVATSSDPTVAGGVELRSVDRG - - - TR IMAVDGR I AMNNPTAAA ITHV VVipPLA-III $\beta$ VCRATSAEPAIFEPVSMRSVDGQ - . - - TRCVA IDGGLAMSNPTAAAITHV VVipPLA-III $\delta$ VCRATTATPSMFKPFSLTSVDGK - - - TSCSAVDGGLVMNNPTAAAVTHV SAAPTY DGGxxANN

Figure 1. Multiple alignments of four grapevine PLA families representing the consensus and conserved motifs. Protein sequences were aligned for each PLA family, separately, applying MAFFT tool. The consensus motifs have been shown in shadow boxes according BLOSUM62. (A) VviPLA1; (B) VviPA-PLA1; (C) VvisPLA2; (D) VvipPLA. 

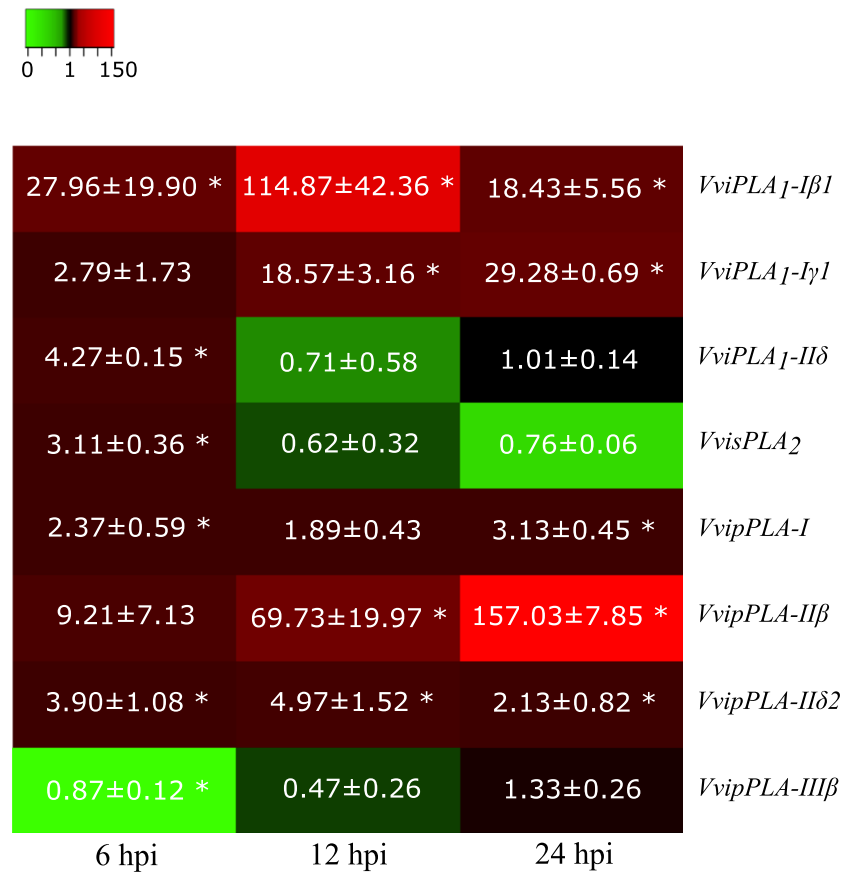

Figure 2. Gene expression profiles in Regent inoculated leaves. For each time point (6,12 and 24 hpi) gene transcripts fold-change relative to controls are represented for VviPLA $A_{1}-I \beta 1$; VviPLA $A_{1} I \gamma 1 ; V v i P L A_{1}-I I \delta$; VvisPLA $A_{2}$; VvipPLA-I; VvipPLA-II $\beta$; VvipPLA-IIS2; VvipPLA-III $\beta$. Fold-change values are relative to expression in mock inoculated leaves. Asterisks indicate significant differences $(\mathrm{p}<0.05)$.

(c) (1) Open Access This article is licensed under a Creative Commons Attribution 4.0 International License, which permits use, sharing, adaptation, distribution and reproduction in any medium or format, as long as you give appropriate credit to the original author(s) and the source, provide a link to the Creative Commons license, and indicate if changes were made. The images or other third party material in this article are included in the article's Creative Commons license, unless indicated otherwise in a credit line to the material. If material is not included in the article's Creative Commons license and your intended use is not permitted by statutory regulation or exceeds the permitted use, you will need to obtain permission directly from the copyright holder. To view a copy of this license, visit http://creativecommons.org/licenses/by/4.0/.

(C) The Author(s) 2019 